\title{
Virus dell'epatite C nei pazienti in trattamento emodialitico in lista per trapianto di rene: l'esperienza dii Padova
}

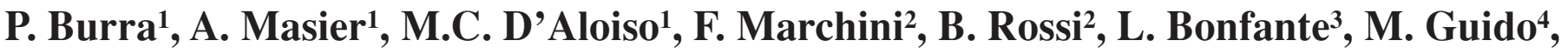 \\ N. Baldan 5 , P. Rigotti ${ }^{5}$ \\ ${ }^{1}$ Gastroenterologia, Dipartimento di Scienze Chirurgiche e Gastroenterologiche; ${ }^{2}$ Nefrologia \\ II; ${ }^{3}$ Nefrologia I; ${ }^{4}$ Anatomia Patologica II; ${ }^{5}$ Clinica Chirurgica III, Azienda Ospedaliera, \\ Università di Padova, Padova
}

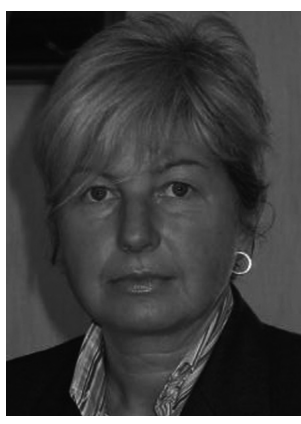

D gennaio 1995 a dicembre 2005 sono stati seguiti presso l'ambulatorio epatologico della Gastroenterologia di Padova 125 pazienti anti$\mathrm{HCV}$ positivi in P. Burra valutazione per trapianto di rene con l'obiettivo di valutare il rischio di progressione della malattia epatica dopo eventuale trapianto di rene.

La valutazione che viene attuata dal nostro Centro prevede di indagare i seguenti parametri bioumorali e strumentali:

- Livelli sierici di ALT. Poiché i livelli sierici di ALT nei pazienti in trattamento emodialitico sono solitamente inferiori a quelli della popolazione sana, per valutare l'ipertransaminasemia nei pazienti $\mathrm{HCV}$ positivi in trattamento emodialitico come limite di normalità è stato considerato il $95^{\circ}$ percentile dei valori sierici di ALT riscontrati nella popolazione in tratta- mento emodialitico senza patologia epatica, come raccomandato da Espinosa (1), che nel gruppo in studio risultava essere di $29 \mathrm{UI} / \mathrm{L}$.

- Anticorpi anti-HCV (EIA II-III o RIBA III) ed HCV-RNA quantitativo (PCR - COBAS AMPLICOR) vengono determinati prima del trapianto di rene e a 1, 6, 12 mesi dal trapianto di rene, in seguito ogni 12 mesi.

- Ecografia epatica: viene effettuata prima del trapianto di rene e ogni 6 mesi dopo il trapianto di rene.

- Esame istologico della biopsia epatica. L'indicazione all'esecuzione della biopsia epatica nei pazienti $\mathrm{HCV}$ positivi in trattamento emodialitico viene posta in base alla positività di HCV-RNA indipendentemente dalla presenza di ipertransaminasemia. La valutazione del danno epatico viene effettuata mediante grading (G0: assenza di attività infiammatoria, G1 attività lieve, G2 attività moderata, G3 attività severa) e staging (S0: assenza di fibrosi, S1: fibrosi portale in assenza di setti, S2: presenza di pochi setti fibrosi, S3: presenza di numerosi setti fibrosi in assenza di cirrosi, S4: cirrosi, secondo la classificazione di Scheuer (2) o di Ishak (3).

In base ai reperti virologici ed istologici ad ogni paziente viene assegnato uno score di progressione della malattia epatica secondo il seguente schema:

rischio lieve: HCV-RNA-, genotipo non- $1 \mathrm{~b}$, epatite cronica lieve $(\mathrm{G}=0-1$; $\mathrm{S}<2$ );

rischio lieve-moderato: $\mathrm{HCV}-\mathrm{RNA}$-/+, genotipo non- $1 \mathrm{~b}$, epatite cronica moderata $(\mathrm{G}=1-2 ; \mathrm{S}<2)$;

rischio moderato: $\mathrm{HCV}-\mathrm{RNA}+$, genotipo $1 \mathrm{~b}$, epatite cronica moderata $(\mathrm{G}=1$ 2; $\mathrm{S}>2$ );

rischio severo-moderato: $\mathrm{HCV}-\mathrm{RNA}+$, genotipo $1 \mathrm{~b}$, epatite cronica severa-moderata $(\mathrm{G}=2-3 ; \mathrm{S}>2)$;

rischio severo: $\mathrm{HCV}-\mathrm{RNA}+$, genotipo $1 \mathrm{~b}$, epatite cronica severa o cirrosi $(\mathrm{G}=3 ; \mathrm{S}>2)$.

I fattori di rischio per l'infezione HCV sono stati identificati solamente in una percentuale limitata di pazienti in trattamento emodialitico (emotrasfusioni, tatuaggi, anamnesi positiva per abuso di sostanze stupefacenti per via endovenosa). Nel gruppo di pazienti valu- 


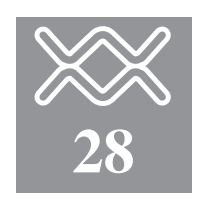

tati, al momento della prima visita ambulatoriale i valori di AST e ALT erano i seguenti: AST 25.02 \pm 19.80 IU/l, ALT $33.35 \pm 29.57 \mathrm{IU} / \mathrm{l}$, e nessun paziente presentava segni clinici o ecografici di ipertensione portale. L'HCV-RNA era positivo in circa l' $80 \%$ dei pazienti, con genotipo $1 \mathrm{~b}$ riscontrato nel $50 \%$ dei pazienti con HCV-RNA positivo.

La valutazione istologica ha permesso di evidenziare epatite cronica lieve nel $25 \%$, moderata nel $37.5 \%$, severa nel $23.2 \%$ e cirrosi nel $14.3 \%$ dei casi.

In base allo score di malattia epatica nei pazienti candidati al trapianto di rene creata dal nostro Centro, descritto per ipotizzare il rischio di progressione della malattia epatica dopo trapianto di rene, questo era lieve nel $36 \%$ dei casi, lieve/moderato nell' $8 \%$, moderato nel $38 \%$, moderato/severo nel $14 \%$ e severo nel $4 \%$ dei casi.

Questi criteri di selezione pre-trapianto hanno permesso di identificare i pazienti che sono stati sottoposti a terapia antivirale prima del trapianto di rene, il $30.4 \%$ dei pazienti sottoposti a biopsia epatica. Nessun episodio di epatite acuta o evidenza clinica di progressione della malattia epatica è stato osservato dopo trapianto di rene in 10 anni di follow-up. In questo gruppo di pazienti la sopravvivenza cumulativa a 10 anni dell'organo e del paziente è risultata rispettivamente di $74 \%$ e $100 \%$.

\section{CONCLUSIONI}

La selezione di pazienti in trattamento emodialitico anti-HCV positivi sulla base di parametri virologici e istomorfologici, con l'identificazione di uno score di rischio di progressione della malattia epatica dopo trapianto di rene ha permesso di individuare i pazienti suscettibili di terapia antivirale e di avere una stima dell'evoluzione della malattia epatica HCV correlata in regime di terapia immunosoppressiva. Nel nostro studio la presenza di anti$\mathrm{HCV}$ positività non ha modificato significativamente la sopravvivenza dell'organo e del paziente a dieci anni dal trapianto di rene rispetto ai dati riportati per i pazienti anti-HCV negativi sottoposti a trapianto di rene.

\section{BIBLIOGRAFIA}

1. Espinosa M, Martin-Malo A, Alvarez de Lara MA, Soriano S, Aljama P. High ALT levels predict viremia in anti-HCV-positive HD patients if a modified normal range of ALT is applied. Clin Nephrol 2000; 54(2):151-6.

2. Scheuer PJ. Classification of chronic viral hepatitis: a need for reassesment. J Hepatol 1991; 13: 372-4.

3. Ishak K, Baptista A, Bianchi L, Callea F, De Groote J, Gudat F, et al. Histological grading and staging of chronic hepatitis. J Hepatol 1995; 22 : 696-9. 